\title{
Accurate Determination, Matrix Effect Estimation, and Uncertainty Evaluation of Three Sulfonamides in Milk by Isotope Dilution Liquid Chromatography-Tandem Mass Spectrometry
}

\author{
Chaonan Han $\mathbb{D}$, ${ }^{1,2}$ Xiuqin Li $\left(\mathbb{D},{ }^{1}\right.$ Hui Jiao $\mathbb{D}^{1},{ }^{1}$ Yan Gao $\mathbb{D}{ }^{1},{ }^{1}$ and Qinghe Zhang $\mathbb{D}^{1}$ \\ ${ }^{1}$ Division of Chemical Metrology and Analytical Science, National Institute of Metrology, Beijing 100029, China \\ ${ }^{2}$ Heilongjiang Green Food Science Research Institute, Harbin 150000, China \\ Correspondence should be addressed to Yan Gao; gaoyan@nim.ac.cn and Qinghe Zhang; zhangqh@nim.ac.cn
}

Received 11 June 2021; Revised 15 August 2021; Accepted 9 September 2021; Published 29 September 2021

Academic Editor: Wei Chen

Copyright ( 2021 Chaonan Han et al. This is an open access article distributed under the Creative Commons Attribution License, which permits unrestricted use, distribution, and reproduction in any medium, provided the original work is properly cited.

Liquid chromatography-tandem mass spectrometry (LC-MS/MS) is the most commonly used method for sulfonamide determination. Its accuracy, however, can be affected by many factors. In this study, sulfadiazine (SDZ), sulfadimidine (SMZ), and sulfadimethoxine (SDM) in milk were selected to investigate an accurate determination method and the potential influencing factors in the use of ultrahigh-performance liquid chromatography-tandem mass spectrometry (UHPLC-MS/MS). Milk samples were extracted by $25 \mathrm{~mL}$ perchloric acid solution $(\mathrm{pH}=2)$ and cleaned up using HLB solid-phase extraction (SPE) cartridges. Four kinds of filters, including PTFE, GHP, nylon, and glass fiber, were compared, and PTFE was selected since it had the best recoveries of target sulfonamides (SAs). Three quantitative methods, including external standard (ES), matrix matching (MM), and isotope dilution mass spectrometry (IDMS), were compared, among which IDMS exhibited the best accuracy. The matrix effect under different mobile phase compositions and of different sample matrices were evaluated and discussed. Ion suppression effects were observed during the determination of all SAs, which got stronger with the increase of the methanol composition percent in the mobile phase. After correction by IDMS, the matrix effect could be neglected. Matrix spiked recoveries at three spiked levels ( $1 \mu \mathrm{g} / \mathrm{kg}, 10 \mu \mathrm{g} / \mathrm{kg}$, and $20 \mu \mathrm{g} / \mathrm{kg}$ ) ranged from $96.8 \%$ to $103.8 \%$ by IDMS. The expanded relative uncertainties were in the range of $2.02 \%$ to $5.75 \%$. The method exhibited wide application range, high accuracy, good stability, and high sensitivity.

\section{Introduction}

Sulfonamides (SAs) are a kind of generic, highly effective, low-toxicity, and low-cost antibacterial agents [1]. The use of SAs as antibacterial synergist can expand the scope and enhance the activity of antibacterial. Therefore, SAs are widely used in milk production for the prevention and treatment of bacterial diseases. However, due to the potential toxicity, excessive intake of SAs may lead to human diseases, such as urinary system damage, digestive disorders, vomiting diarrhea, hemolytic anemia, drug-resistant strains, human immunity reduction, and tumor tendency $[2,3]$.

Therefore, many countries and regions have issued regulations on maximum residue limits (MRL) for SAs in food and feed. It is stipulated in the International Codex Alimentarius
Commission (CAC) that the total amount of SAs in food and feed shall not exceed $100 \mu \mathrm{g} / \mathrm{kg}$ [4]. The United Nations Food and Agriculture Organization (FAO) stipulates a residue limit for SAs in animal food at $100 \mathrm{ng} / \mathrm{mL}$ [5]. According to the European Union (EU) regulation, single SAs concentration in milk and meat should not exceed $25 \mu \mathrm{g} / \mathrm{kg}$, and the total amount should not exceed $100 \mu \mathrm{g} / \mathrm{kg}$ [6]; China's Ministry of Agriculture and Rural Affairs stipulates that the total concentration of SAs in milk should be lower than $100 \mathrm{ng} / \mathrm{mL}$, and the MRL of sulfamethazine is $25 \mu \mathrm{g} / \mathrm{L}$ [7].

At present, the detection methods of SAs residues mainly include microbial detection [8], fluorescence spectrophotometry [9], immunoassay [10], and high-performance liquid chromatography coupled mass spectrometry (HPLCMS). However, the sensitivity and selectivity were limited. 
Liquid chromatography coupled to tandem mass spectrometry (LC-MS/MS) $[11,12]$ is a highly sensitive, specific, and reliable tool for contaminants detection in food and has become the most universal approach for multianalyte analysis. It is applied in an expanding field of food analysis, particularly in multiresidue detection $[13,14]$. However, the complex food matrix (such as carbohydrates, proteins, or fats) can induce ion suppression or enhancement of the target analytes, which may hamper the accuracy of mass spectrometric quantification [15-18]. Matrix effect (ME) cannot be neglected in LC-MS/MS analysis, which makes the extraction and clean-up processes challenging [19-22]. Strategies have been developed to minimize or eliminate the MEs, such as improving chromatographic selectivity to avoid coelution, mobile phase modifiers, dilution, and efficient clean-up [23-25]. Although these approaches were claimed to be effective for chosen mode analytes, there are inherent drawbacks for further extension [26, 27]. With identical chemical and chromatographic properties compared with the target compound, isotope labeled internal standards can compensate for matrix effects. Therefore, the isotope dilution mass spectrometry (IDMS) method exhibits high accuracy and repeatability for the quantitative analysis of organic compounds in complex matrices, which could overcome the difficulty in the correction of recovery and the influence of inject volume, mobile phase, and instrument fluctuation during the sample detection [28-34].

The accuracy and reliability of the method are crucial in veterinary drugs analysis. In this study, three kinds of SAs with different polarities, SDZ (pKow=0.34), SMZ ( $\mathrm{pKow}=-0.76)$, and SDM (pKow $=-1.17)$ (pKow obtained from KowWin software), were selected. The experimental conditions were optimized and discussed thoroughly. The extraction and clean-up methods were optimized. Four kinds of filters were compared. Three quantitative methods, external standard (ES), matrix matching (MM), and isotope dilution mass spectrometry (IDMS), were compared. Matrix effects were evaluated, and the potential influencing factors were discussed. Moreover, the uncertainty of the method was estimated. The developed method was validated for accuracy and precision through the matrix spiked experiment, intra-/interday variation, limits of detection (LOD), limits of quantification (LOQ), linearity, and uncertainties.

\section{Materials and Methods}

2.1. Materials and Reagents. The certified reference materials (CRMs) of sulfadiazine (SDZ; GBW (E) 081146), sulfadimidine (SMZ; GBW (E) 081145), and sulfadimethoxine (SDM; GBW (E) 061416) were obtained from the National Institute of Metrology, China (Beijing, China). The isotope standards $\left({ }^{13} \mathrm{C}_{6}\right.$-sulfadiazine, ${ }^{13} \mathrm{C}_{6}$-sulfadimidine, and ${ }^{13} \mathrm{C}_{6}$ sulfadimethoxine) were purchased from TRC (Toronto, Canada). Formic acid, methanol, and water (HPLC-MS grade) were purchased from Thermo Fisher Scientific (Waltham, MA, USA). Perchloric acid (analytical grade) was purchased from XiYa chemical reagents company (Shanxi, China). Oasis ${ }^{\circledR}$ HLB SPE cartridges $(6 \mathrm{cc}, 150 \mathrm{mg}$ ) were purchased from Waters (Milford, MA, USA).
Stock solutions were made for individual sulfonamides at the concentration of $1000 \mu \mathrm{g} / \mathrm{kg}$ in methanol and stored away from light at $4^{\circ} \mathrm{C}$. For linearity studies, the samples were freshly prepared using appropriate dilution of the stock solution with initial LC mobile phase (methanol-water; 15 : $85 ; \mathrm{v}: \mathrm{v}$, containing $0.2 \%$ formic acid). Standard solutions of three sulfonamides mixture were prepared in the range of 0.1 to $50 \mu \mathrm{g} / \mathrm{kg}$ with isotopic internal standards at $10 \mu \mathrm{g} / \mathrm{kg}$. Spiked milk samples were prepared at three concentrations of 1,10 , and $20 \mu \mathrm{g} / \mathrm{kg}$, respectively. All standard solutions were stored in amber glass bottles at $4^{\circ} \mathrm{C}$.

2.2. Milk Samples. Skim and whole-fat milk samples of Yili (China), Arla (Germany), and LVLINB (Austria) were purchased from supermarket. The nutrient contents of milk are shown in Table S1.

2.3. Sample Preparation. A previously homogenized milk sample $(1.00 \mathrm{~g})$ was weighed into a $50 \mathrm{~mL}$ centrifuge tube. $10 \mathrm{ng}$ internal standard (100 ng/g working solutions) was added. Then, $25 \mathrm{~mL}$ perchloric acid solution $(\mathrm{pH}=2)$ was added to each tube, and the mixture of each tube was vortexmixed for $1 \mathrm{~min}$ and then extracted by ultrasonic for $10 \mathrm{~min}$.

HLB solid-phase extraction cartridge $(6 \mathrm{cc}, 150 \mathrm{mg})$ was preconditioned with $5 \mathrm{~mL}$ methanol and $5 \mathrm{~mL}$ perchloric acid solution $(\mathrm{pH}=2)$ sequentially at a flow rate of $1 \mathrm{~mL} /$ min. The extraction solution was gradually loaded to the HLB cartridge. Then, the centrifuge tube was washed with $5 \mathrm{~mL}$ perchloric acid solution and the wash solution was also loaded to the cartridge. The cartridge was washed with $5 \mathrm{~mL}$ water and then dried under vacuum for $1 \mathrm{~min}$, and the SAs were eluted with $3 \mathrm{~mL}$ methanol at a flow rate of $1 \mathrm{~mL} / \mathrm{min}$. The elution liquid was evaporated under a gentle nitrogen stream at $40^{\circ} \mathrm{C}$ until the remaining amount was around $200 \mu \mathrm{L}$. The residual liquid was reconstituted with $1 \mathrm{~mL}$ methanol-water $(15: 85 ; \mathrm{v}: \mathrm{v}$, containing $0.2 \%$ formic acid), vortex-mixed for $1 \mathrm{~min}$, and filtered through a $0.22 \mu \mathrm{m}$ PTFE filter into a glass LC vial for LC-MS/MS analysis.

2.4. UHPLC-MS/MS Conditions. UHPLC-/MS/MS system, which consisted of a LC30AD liquid chromatography (Shimadzu Corp., Japan) and QTRAP 5500 mass spectrometry (SCIEX Corp., CA, America), was used for SAs analysis. The separation of SAs was achieved on an Acquity UPLC $\mathrm{CSH}^{\mathrm{TM}} \mathrm{C} 18$ column $(100 \times 3.0 \mathrm{~mm}, 1.7 \mu \mathrm{m}$ particle size; Waters, USA). A gradient LC elution method was employed by $0.2 \%$ formic acid aqueous solution as mobile phase $\mathrm{A}$ and acetonitrile (containing $0.2 \%$ formic acid) as mobile phase B. The gradient elution was carried out as follows: $15 \% \mathrm{~B}$ maintained for $3 \mathrm{~min}$; increased to $35 \% \mathrm{~B}$ linearly in $2 \mathrm{~min}$ and then increased to $100 \% \mathrm{~B}$ in $4 \mathrm{~min}$ and maintained for $1 \mathrm{~min}$; changed to $15 \% \mathrm{~B}$ and maintained for $2 \mathrm{~min}$. The injection volume was $5 \mu \mathrm{L}$. The flow rate was set at $0.25 \mathrm{~mL} / \mathrm{min}$, and the column temperature was set at $38^{\circ} \mathrm{C}$.

The QTRAP 5500 mass spectrometer equipped with an electrospray ionization (ESI) source was performed in positive ionization multiple-reaction monitoring (MRM) 
mode. The ion source temperature (TEM) was set at $600^{\circ} \mathrm{C}$, and ion spray voltage (IS) was set at $5.5 \mathrm{kV}$. Ion source gas 1 (GS1) and ion source gas 2 (GS2) were used as the drying and nebulizer gases at a back pressure of $60 \mathrm{psi}$ and $65 \mathrm{psi}$, respectively. Curtain gas (CUR) was 25 psi. $\mathrm{N}_{2}$ was used for all the gases. Parameters such as declustering potential (DP) and collision energy (CE) for the analyte are shown in Table S2.

2.5. Matrix Effect Estimation. The ME factors were investigated by comparing the signal intensity and spiked quality of matrix-matched standard solution with that of the standard solution at the same concentrations. If the peak areas and mass obtained in neat solution standards were set as $A$ and $m$ and the corresponding peak areas for standards spiked after extraction into milk extracts as $A^{\prime}$ and $m^{\prime}$, the ME factors can be calculated as follows [35]:

$$
\mathrm{ME}=\frac{A^{\prime} / m^{\prime}}{A / m} .
$$

ME factor $<1$ indicated ion suppression effect and ME factor $>1$ indicated ion enhancement effect.

2.6. Validation of the Method. The LOD and LOQ were defined as $3 \times$ signal-to-noise ratio $(\mathrm{S} / \mathrm{N})$ and $10 \times \mathrm{S} / \mathrm{N}$, respectively. To determine the linearity of the method, standard solutions of three sulfonamides mixture were prepared at the concentration of $0.1,0.5,1,10,20$, and $50 \mu \mathrm{g} / \mathrm{kg}$ with isotopic internal standards at the concentration of $10 \mu \mathrm{g} / \mathrm{kg}$.

To evaluate the recovery and precision, blank milk samples were spiked with three sulfonamides mixture standard solution at three concentrations of 1,10 , and $20 \mu \mathrm{g} /$ $\mathrm{kg}$, respectively. The precision was evaluated as intraday and interday by measuring the corresponding relative standard deviations (RSDs) at two spiked concentrations $(1 \mu \mathrm{g} / \mathrm{kg}$ and $20 \mu \mathrm{g} / \mathrm{kg}$ ) on six sequential runs by analyzing six replicates. Intraday precision (the so-called repeatability) was measured on a single day, whereas interday precision was evaluated on six consequent days.

\section{Results and Discussion}

3.1. Optimization of UHPLC-MS/MS Conditions. Acquity UPLC $\mathrm{CSH}^{\mathrm{TM}} \mathrm{C} 18$ column $(100 \times 3.0 \mathrm{~mm}, 1.7 \mu \mathrm{m})$ and XTerra MS C18 $(100 \times 2.1 \mathrm{~mm}, 3.5 \mu \mathrm{m})$ column were compared under the same experimental condition. The results showed that all compounds can be separated on both columns, and the target compounds had shorter retention time on XTerra MS C18 column. All the compounds had higher signal-to-noise ratio values in the Acquity UPLC C18 $\mathrm{CSH}^{\mathrm{TM}}$ column. Finally, the Acquity UPLC CSH ${ }^{\mathrm{TM}} \mathrm{C} 18$ column was selected for the experiment.

Methanol and acetonitrile with different concentrations of formic acid $(0.1 \%$ and $0.2 \%)$ were compared, respectively. For methanol and acetonitrile with the same formic acid concentration $(0.1 \%$ formic acid), the responses of SDZ and SDM were about $20 \%$ and $5 \%$ higher by using methanol, while the responses of SMZ were similar. The response of
SMZ was about $20 \%$ higher on using methanol with $0.2 \%$ formic acid than that on using methanol with $0.1 \%$ formic acid. Methanol containing $0.2 \%$ formic acid was selected for the following experiments, as this condition provided the highest response. The chromatography of 3 SAs is illustrated in Figure S1.

The optimization of parameters can directly affect the sensitivity and accuracy of mass spectrometry. To obtain maximum sensitivity for the identification and detection of the three sulfonamides, compound-dependent parameters such as cone voltage and collision energy were optimized by direct infusion of different standard solutions at $100 \mu \mathrm{g} / \mathrm{kg}$ and at a flow rate of $7 \mu \mathrm{L} / \mathrm{min}$ using the built-in syringe pump directly connected to the interface. Precursor ions and product ions were determined, and the transition with the best sensitivity was selected for quantification, whereas an additional transition was acquired for confirmation (Table S2).

3.2. Optimization of Pretreatment Method. Based on the national standard method (GB/T 22966-2008), the pretreatment method was improved and optimized. During the experiment, it was found that the filter membrane had a certain effect on the recovery rates of the analytes, so the selection of filter membrane was mainly optimized. This has rarely been discussed in previous studies.

Four kinds of filter membranes, including PTFE $(0.45 \mu \mathrm{m}, 25 \mathrm{~mm})$, GHP $(0.2 \mu \mathrm{m}, 13 \mathrm{~mm})$, nylon $(0.2 \mu \mathrm{m}$, $15 \mathrm{~mm})$, and glass fiber $(1.0 \mu \mathrm{m}, 15 \mathrm{~mm})$ filters, were chosen to study the adsorption behavior by comparing the absolute recoveries of standard solution. The recovery is shown in Figure 1.

The adsorption of target compounds through PTFE filter was very low, and the recoveries ranged from $98.4 \%$ to $100.5 \%$. It was obviously observed that the recovery of SMZ was only $65.7 \%$ after filtering through GHP filter; the recoveries of SDZ and SDM were $94.9 \%$ and $97.2 \%$, respectively. Nylon filter had apparent adsorption for three SAs, with the recoveries ranging from $72.1 \%$ to $89.6 \%$, and glass fiber filter recoveries were between $98.0 \%$ and $99.2 \%$. Therefore, the PTFE filter was selected in this study because it has the lowest adsorption for the target compounds.

3.3. Comparison of Quantitation Methods. The choice of quantitation method had a significant impact on the accuracy of the results. For the three compounds, the quantitation methods were compared using the skimmed milk of Yili under gradient elution conditions as shown in Figure 2. The recoveries by external standard method ranged from $17.8 \%$ to $61.2 \%$, which were the lowest of all modes, and the difference between target compounds was large. The external standard method was greatly affected by the matrix, having a serious effect on the accuracy and reliability of the results. Blank sample matrix was applied to compensate for matrix effect by using a matrix matching method. Although the recoveries of matrix matching method ranged from $80.9 \%$ to $95.2 \%$, which were better than those of external standard method, the recovery of individual target compound was still unsatisfactory. Besides, the ME factors of different milk 


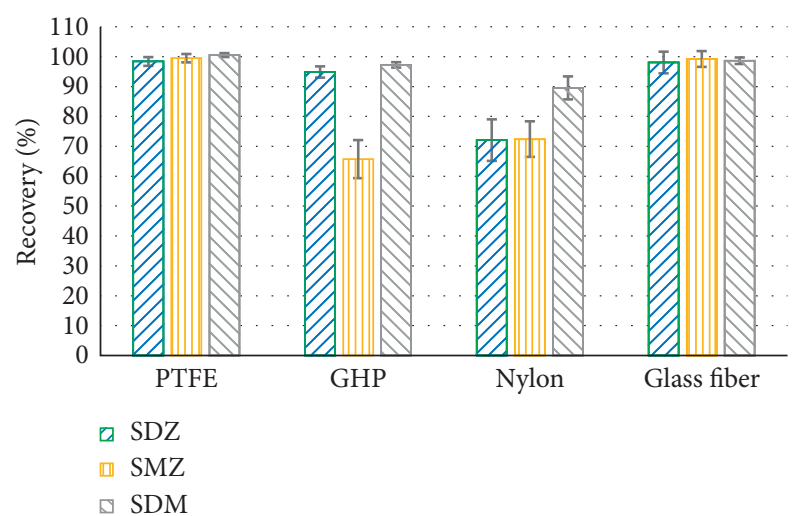

Figure 1: Absolute recoveries of filter after filtration of standard solution $(n=3)$.

samples might be different, and it was very time-consuming and difficult to obtain the same blank matrix samples. The recoveries of IDMS method ranged from $99.7 \%$ to $100.7 \%$. Due to similar physical and chemical properties between the isotope labeled internal standard and the target compound, their behaviors in the process of pretreatment and instrumental analysis were basically consistent, which greatly reduces the interference of the matrix effect. Moreover, the quantitative analysis by IDMS method had the lowest deviation and the best stability among the three methods.

Recoveries of IDMS method were the best under the two isocratic conditions and the gradient elution condition (as shown in Figure S2). Besides, the results of IDMS were similar under the three elution conditions while the recoveries varied by MM and ES, especially ES. The results indicated that IDMS method had a higher accuracy and better stability than MM and ES, leading to an extensive range of application. It was one of the most commonly used methods to detect trace components in complex matrix.

The quantitation methods for six different milk samples of skim and whole-fat milk purchased from local market were also compared. The recoveries of six matrix samples were analyzed by ES, MM, and IDMS, respectively. The results are shown in Table S3. External standard and matrix matching method were used to calculate the recovery, which was very unstable and can be greatly affected by the matrix. The recovery rates of IDMS method ranged from $96.6 \%$ to $102.2 \%$, which can effectively reduce the matrix effect, exhibiting a high stability and wide applicability. The results indicated that the IDMS method can provide a stable and reliable method for the detection and analysis of SAs in milk.

3.4. Estimation of Matrix Effects. Although some interfering components can be eliminated during the extraction, clean-up, and chromatographic separation process, analytical errors and inaccurate results still existed due to the interfering substances in complex matrix. The consequences of matrix effect were the over- or underestimation of the actual concentration of analytes present in samples, affecting both trueness and precision of the analytical method. The methods used to evaluate the matrix effects in LC-MS/MS analysis included postcolumn

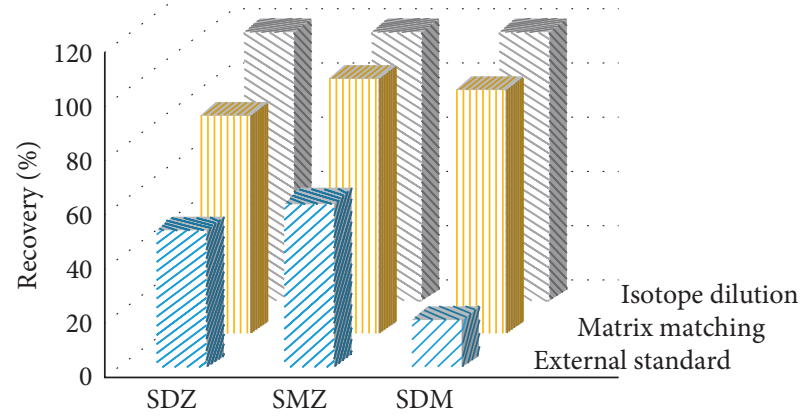

FIgURE 2: The recoveries of compounds in different quantitative modes with gradient elution.

injection and postextraction addition $[16-18,36]$. The method of postextraction addition can quantify the matrix effect, and it was widely used in the verification procedure.

First, the matrix effects of analytes in different milk matrix samples were compared (Table 1). The results showed that all compounds showed ion suppression effect in different milk samples. The ME factors for SDZ, SMZ, and SDM were in the following ranges: from 0.63 to 0.87 ; from 0.64 to 0.73 ; and from 0.18 to 0.30 , respectively. There was a little difference in the matrix effect of different brands of skim-fat milk or whole-fat milk. However, the ME factors of skim-fat milk samples were lower than those of whole-fat milk, which indicated that the ion suppression effects of skim-fat milk were greater. The reason was unclear, and it may be related to the composition content in milk samples.

The ME factors (Yili skim-fat milk) of the target analytes at four liquid chromatography elution conditions were calculated, respectively. As shown in Figure 3, the ME factors of three compounds were lower than 1 under different phase compositions, which indicated ion suppression effect of target compounds in all mobile phase conditions. The ion suppression effects of SDZ and SMZ were lower under gradient condition than isocratic, and the ME factors in isocratic condition of $50 \%$ methanol were minimum. With the increase of the methanol composition percent in the mobile phase, the inhibitory effect got stronger, inferring that coeluting components in the matrix (interferents) under high methanol composition may get better atomization effect and higher ionization efficiency. The enhancement of the competitive ion lead to stronger ion suppression effect on the target compounds. Moreover, there may be more coeluting matrix with higher methanol composition.

After the correction by isotope labeled internal standards, the MEs for SDZ, SMZ and SDM were in the following ranges: from 0.937 to 1.001 ; from 0.973 to 0.999 ; from 1.006 to 1.021 , respectively, indicating that IDMS could compensate for matrix effect to a large content.

3.5. Uncertainty Evaluation. The uncertainty of the IDUHPLC-MS/MS analysis was evaluated by the combination of individual uncertainty including purity of SAs CRMs 
TABle 1: The matrix effect factors of the target analytes in six milk samples $(n=3)$.

\begin{tabular}{lccccccc}
\hline & & Yili & & \multicolumn{2}{c}{ Arla } & \multicolumn{2}{c}{ LVINB } \\
& Skim & & Whole & Skim & Whole & Skim & Whole \\
\hline SDZ & 0.63 & 0.81 & 0.86 & 0.87 & 0.83 & 0.87 \\
SMZ & 0.64 & & 0.73 & 0.67 & 0.66 & 0.68 & 0.70 \\
SDM & 0.20 & 0.24 & 0.18 & 0.25 & 0.18 & 0.30 \\
\hline
\end{tabular}

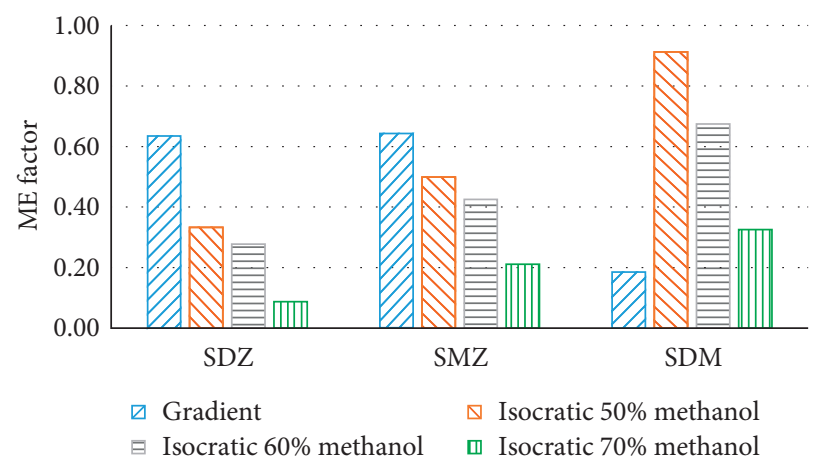

Figure 3: The matrix effect factors of the target analytes with four kinds of mobile phase elution conditions $(n=3)$.

$\left(u_{\text {rel, }}\right)$, balance weighing $\left(u_{\text {rel,w }}\right.$; including weighing of standards and standard solutions, isotope labeled standards and standard solutions, and milk samples), and the method precision $\left(u_{\text {rel, rep }} ; s / \sqrt{n}\right)$.

The uncertainty from balance weighing referred to weights of CRMs of pure SAs and isotope labeled SAs, solvents, standard solutions, milk samples, and isotope labeled SAs solutions. The uncertainty of balance was evaluated as rectangular distribution. The standard uncertainty of balance weighting $\left(\mathcal{u}_{\mathrm{b}}\right)$ is the combination of repeatability and weighting tolerance. The sample weighing is obtained by two times weighing operation, and each weight is an independent observation.

When a different candidate is weighed for the analysis $\left(w_{i}\right)$, the relative uncertainty is determined by formula (2). The uncertainty from balance weighing $u_{\text {rel, w }}$ is calculated by combining all uncertainties from weighing using formula (3):

$$
\begin{aligned}
& u_{\text {rel, } \mathrm{i}}=\frac{u_{\mathrm{b}}}{w_{i}}, \\
& u_{\mathrm{rel}, \mathrm{i}}=\sqrt{\sum u_{\mathrm{rel}, \mathrm{i}}^{2}}
\end{aligned}
$$

The uncertainty of method precision was in the range of $0.25 \%-2.46 \%$. The combined relative standard uncertainty $(u)$ was calculated by formula (4). The expanded relative uncertainties $(U)$ with a coverage factor of $2(k=2)$ was calculated by formula (5):

$$
\begin{aligned}
& u=\sqrt{u_{\text {rel,p }}^{2}+u_{\text {rel, } \mathrm{w}}^{2}+u_{\text {rel,rep }}^{2},} \\
& U=2 \times u .
\end{aligned}
$$

The individual uncertainty, relative uncertainties, and expanded relative uncertainties of three spiked levels are listed in Table 2. The relative uncertainties of three SAs were
TABLE 2: Estimated individual uncertainty, relative uncertainty $(u)$, and expanded relative uncertainty $(U, k=2)$ of three spiked levels (\%).

\begin{tabular}{lcccc}
\hline Spiked levels & Uncertainty & SDZ & SMZ & SDM \\
\hline & $u_{\text {rel, }}$ & 2.01 & 1.00 & 1.01 \\
& $u_{\text {rel,w }}$ & 1.80 & 1.80 & 1.80 \\
$1 \mu \mathrm{g} / \mathrm{kg}(n=6)$ & $u_{\text {rel,rep }}$ & 0.99 & 0.28 & 1.00 \\
& $u$ & 2.87 & 2.08 & 2.30 \\
& $U$ & 5.75 & 4.16 & 4.59 \\
\hline & $u_{\text {rel, p }}$ & 2.01 & 1.00 & 1.01 \\
& $u_{\text {rel,w }}$ & 0.18 & 0.18 & 0.18 \\
$10 \mu \mathrm{g} / \mathrm{kg}(n=6)$ & $u_{\text {rel,rep }}$ & 0.58 & 0.26 & 0.17 \\
& $u$ & 2.10 & 1.05 & 1.04 \\
& $U$ & 4.20 & 2.10 & 2.08 \\
\hline & $u_{\text {rel, p }}$ & 2.01 & 1.00 & 1.01 \\
& $u_{\text {rel, }}$ & 0.09 & 0.09 & 0.09 \\
$20 \mu \mathrm{g} / \mathrm{kg}(n=6)$ & $u_{\text {rel,rep }}$ & 0.42 & 0.10 & 0.38 \\
& $u$ & 2.06 & 1.01 & 1.08 \\
& $U$ & 4.11 & 2.02 & 2.16 \\
\hline
\end{tabular}

in the range of $1.01 \%$ to $2.87 \%$; the expanded relative uncertainties were in the range of $2.02 \%$ to $5.75 \%$.

3.6. Method Validation. Table S4 summarizes the calibration equations, linearity, correlation coefficient, LOD, and LOQ with the developed UHPLC-MS/MS method. All studied SAs presented good linearity in a wide range of concentrations from $0.1 \mu \mathrm{g} / \mathrm{kg}$ to $50 \mu \mathrm{g} / \mathrm{kg}$. Correlation coefficients $\left(R^{2}\right)$ were higher than 0.999 , suggesting a good linearity of the method. LOD and LOQ were estimated as $3 \times \mathrm{S} / \mathrm{N}$ and $10 \times \mathrm{S} / \mathrm{N}$, respectively. LOD ranged from $0.018 \mu \mathrm{g} / \mathrm{kg}$ to $0.075 \mu \mathrm{g} / \mathrm{kg}$, while LOQ ranged from $0.029 \mu \mathrm{g} / \mathrm{kg}$ to $0.166 \mu \mathrm{g} / \mathrm{kg}$. The low LOQ obtained by this method allowed the quantification at concentrations lower than the MRL stipulated in current legislation. Recovery was evaluated at three spiked levels $(1 \mu \mathrm{g} / \mathrm{kg}$, $10 \mu \mathrm{g} / \mathrm{kg}$, and $20 \mu \mathrm{g} / \mathrm{kg}$ ), as shown in Table 3 . Six replicates of 
TABLE 3: Recoveries of SDZ, SMZ, and SDM in the different spiked levels and the method precisions at two different concentrations combined with LC-MS/MS method of the three sulfonamides from milk samples $(n=6)$.

\begin{tabular}{lccccccc}
\hline Compounds & \multicolumn{3}{c}{ Recovery (\%) } & \multicolumn{2}{c}{$\begin{array}{c}\text { Intraday precision RSD } \\
(\%)\end{array}$} & \multicolumn{2}{c}{$\begin{array}{c}\text { Interday precision RSD } \\
(\%)\end{array}$} \\
& $1 \mu \mathrm{g} / \mathrm{kg}$ & $10 \mu \mathrm{g} / \mathrm{kg}$ & $20 \mu \mathrm{g} / \mathrm{kg}$ & $1 \mu \mathrm{g} / \mathrm{kg}$ & $20 \mu \mathrm{g} / \mathrm{kg}$ & $1 \mu \mathrm{g} / \mathrm{kg}$ & $20 \mu \mathrm{g} / \mathrm{kg}$ \\
\hline SDZ & 99.2 & 100.6 & 96.8 & 2.43 & 1.03 & 0.45 \\
SMZ & 98.2 & 101.2 & 100.6 & 0.69 & 0.25 & 0.90 \\
SDM & 100.7 & 100.9 & 103.8 & 2.69 & 0.74 & 1.02 \\
\hline
\end{tabular}

spiked samples at the three concentration levels were prepared. For all compounds, satisfactory recoveries (96.8\%$103.8 \%$ ) were achieved. Intraday and interday precisions were evaluated at $1 \mu \mathrm{g} / \mathrm{kg}$ and $10 \mu \mathrm{g} / \mathrm{kg}$ spiked levels. For all analytes, the repeatability RSDs for intraday precision were within the range of $0.25-2.69 \%$ and those of interday precision were less than $1.40 \%$, indicating that the method was accurate and precise. The sensitivity and precision of the three SAs in this method were better than the Ministry of Agriculture Announcement No.781-12-2006 standard [37].

\section{Conclusions}

An ID-UHPLC-MS/MS method has been developed and validated for the accurate determination of sulfonamides in milk. After solid-phase extraction and filtration, the sample was analyzed by UHPLC-MS/MS system. The matrix effects were evaluated and discussed. The results showed that there were obvious differences in matrix effect with different mobile phases and different milk matrices. IDMS was used for the quantitation since it could effectively reduce the influence of matrix and other factors. The optimized method was fully validated through estimation of recovery, linearity, LOD/LOQ, and intra-/interday reproducibility, exhibiting good sensitivity, accuracy, and precision. The uncertainty estimation indicated that the developed method has proper metrological quality as reference method in not only daily SAs determination but also value assignment of related CRMs.

\section{Data Availability}

Data are available in the supplementary information file.

\section{Conflicts of Interest}

The authors declare that there are no conflicts of interest regarding the publication of this study.

\section{Acknowledgments}

This study was supported by the National Key Research and Development Program of China (2016YFF0201106) and the Fundamental Research Funds for Central Public Welfare Scientific Research Institutes (21-AKYZZ2117).

\section{Supplementary Materials}

Table S1: nutrients in milk (per $100 \mathrm{~mL}$ ). Table S2: the optimal parameters of multiple-reaction monitoring of SAs.
Table S3: recoveries of different quantitative modes including external standard, matrix matching, and isotope dilution in six milk samples $(n=3)$. Table S4: linearity, LOD, and LOQ of the method. Figure S1: chromatography of three SAs. Figure S2: recoveries of different quantitative analysis modes of three target compounds in different elution conditions. (Supplementary Materials)

\section{References}

[1] Q. Shen, R. Jin, J. Xue, Y. Lu, and Z. Dai, “Analysis of trace levels of sulfonamides in fish tissue using micro-scale pipette tip-matrix solid-phase dispersion and fast liquid chromatography tandem mass spectrometry," Food Chemistry, vol. 194, pp. 508-515, 2016.

[2] L. Wang, B. Yang, X. Zhang, and H. Zheng, "Novel twodimensional liquid chromatography-tandem mass spectrometry for the analysis of twenty antibiotics residues in dairy products," Food Analytical Methods, vol. 10, no. 6, pp. 2001-2010, 2017.

[3] F. L. D. Pontes, J. C. Gasparetto, T. M. G. de Francisco et al., "Development and validation of a multiclass method for the analysis of veterinary drug residues in eggs using liquid chromatography-tandem mass spectrometry," Food Analytical Methods, vol. 10, no. 4, pp. 1063-1077, 2017.

[4] Codex Alimentarius Commission, Maximum Residue Limits for Veterinary Drugs in Foods, Updated as at the 35th Session of the Codex Alimentarius Commission, CAC/MRL, 2012, http://www.fao.org/fao-who-codexalimentarius.

[5] L. Jank, M. T. Martins, J. B. Arsand et al., "Liquid chromatography-tandem mass spectrometry multiclass method for 46 antibiotics residues in milk and meat: development and validation," Food Analytical Methods, vol. 10, no. 7, pp. 2152-2164, 2017.

[6] Commission Regulation (EU) No. 37/2010 of 22 December 2009, On Pharmacologically Active Substances and Their Classification Regarding Maximum Residue Limits in Foodstuffs of Animal Origin, http://citeseerx.ist.psu.edu/ showciting? cid $=18868500$.

[7] Ministry of Agriculture and Rural Affairs of the People's Republic of China, GB 31650-2019, The Maximum Veterinary Drug Residues in Veterinary Drug Residues.

[8] H.-H. Chung, J.-B. Lee, Y.-H. Chung, and K.-G. Lee, “Analysis of sulfonamide and quinolone antibiotic residues in Korean milk using microbial assays and high performance liquid chromatography," Food Chemistry, vol. 113, no. 1, pp. 297-301, 2009.

[9] R. Dez, L. Sarabia, and M. C. Ortiz, "Rapid determination of sulfonamides in milk samples using fluorescence spectroscopy and class modeling with $n$-way partial least squares," Analytica Chimica Acta, vol. 585, pp. 350-360, 2007.

[10] D. V. Yaroshenko and L. A. Kartsova, "Matrix effect and methods for its elimination in bioanalytical methods using 
chromatography-mass spectrometry," Journal of Analytical Chemistry, vol. 69, no. 4, pp. 311-317, 2014.

[11] C. L. Chitescu, A. I. Nicolau, A. Csuma, and C. Moisoiu, "Simultaneous analysis of four sulfonamides in chicken muscle tissue by HPLC," Food Additives \& Contaminants: Part A, vol. 28, no. 8, pp. 1013-1020, 2011.

[12] J. Ma, S. Fan, L. Sun, L. He, Y. Zhang, and Q. Li, "Rapid analysis of fifteen sulfonamide residues in pork and fish samples by automated on-line solid phase extraction coupled to liquid chromatography-tandem mass spectrometry," Food Science and Human Wellness, vol. 9, no. 4, pp. 363-369, 2020.

[13] C. Nebot, P. Regal, J. M. Miranda, C. Fente, and A. Cepeda, "Rapid method for quantification of nine sulfonamides in bovine milk using HPLC/MS/MS and without using SPE," Food Chemistry, vol. 141, no. 3, pp. 2294-2299, 2013.

[14] J. Kang, S.-J. Park, H.-C. Park et al., "Multiresidue screening of veterinary drugs in meat, milk, egg, and fish using liquid chromatography coupled with ion trap time-of-flight mass spectrometry," Applied Biochemistry and Biotechnology, vol. 182, no. 2, pp. 635-652, 2017.

[15] P. Kaczyński, "Clean-up and matrix effect in LC-MS/MS analysis of food of plant origin for high polar herbicides," Food Chemistry, vol. 230, pp. 524-531, 2017.

[16] Y. Wang, S. Li, F. Zhang et al., "Study of matrix effects for liquid chromatography-electrospray ionization tandem mass spectrometric analysis of 4 aminoglycosides residues in milk," Journal of Chromatography A, vol. 1437, pp. 8-14, 2016.

[17] X. Q. Li, Z. Yang, Q. H. Zhang, and H. M. Li, "Evaluation of matrix effect in isotope dilution mass spectrometry based on quantitative analysis of chloramphenicol residues in milk powder," Analytica Chimica Acta, vol. 807, pp. 75-83, 2014.

[18] H. Li, J. Wu, C. Chen, W. Xin, and W. Zhang, "Simultaneous determination of 74 pesticide residues in Panax notoginseng by QuEChERS coupled with gas chromatography tandem mass spectrometry," Food Science and Human Wellness, vol. 10, no. 2, pp. 241-250, 2021.

[19] P. Svoboda, H. Vlčková, and L. Nováková, “Development and validation of UHPLC-MS/MS method for determination of eight naturally occurring catechin derivatives in various tea samples and the role of matrix effects," Journal of Pharmaceutical and Biomedical Analysis, vol. 114, pp. 62-70, 2015.

[20] M. Yadav, V. Trivedi, V. Upadhyay et al., "Comparison of extraction procedures for assessment of matrix effect for selective and reliable determination of atazanavir in human plasma by LC-ESI-MS/MS," Journal of Chromatography B, vol. 885-886, pp. 138-149, 2012.

[21] P. Svoboda, D. Sander, K. Plachká, and L. Nováková, "Development of matrix effect-free MISPE-UHPLC-MS/MS method for determination of lovastatin in Pu-erh tea, oyster mushroom, and red yeast rice," Journal of Pharmaceutical and Biomedical Analysis, vol. 140, pp. 367-376, 2017.

[22] P. Yang, J. S. Chang, J. W. Wong et al., "Effect of sample dilution on matrix effects in pesticide analysis of several matrices by liquid chromatography-high-resolution mass spectrometry," Journal of Agricultural and Food Chemistry, vol. 63, pp. 5169-5177, 2015.

[23] W. X. Zhu, J. Z. Yang, W. Wei, Y.-f. Liu, and S.-s. Zhang, "Simultaneous determination of 13 aminoglycoside residues in foods of animal origin by liquid chromatography-electrospray ionization tandem mass spectrometry with two consecutive solid-phase extraction steps," Journal of Chromatography A, vol. 1207, pp. 29-37, 2008.

[24] R. Pascoe, J. P. Foley, and A. I. Gusev, "Reduction in matrixrelated signal suppression effects in electrospray ionization mass spectrometry using on-line two-dimensional liquid chromatography," Analytical Chemistry, vol. 73, no. 24, pp. 6014-6023, 2001.

[25] C. Ferrer, A. Lozano, A. Agüera, A. J. Girón, and A. R. Fernández-Alba, "Overcoming matrix effects using the dilution approach in multiresidue methods for fruits and vegetables," Journal of Chromatography A, vol. 1218, no. 42, pp. 7634-7639, 2011.

[26] W. Guo, G. Li, Y. Yang, C. Yang, L. Si, and J. Huang, "LC-MS/ MS analysis of pramipexole in mouse plasma and tissues: elimination of lipid matrix effects using weak cation exchange mode based solid-phase extraction," Journal of Chromatography $B$, vol. 988, pp. 157-165, 2015.

[27] O. González, M. Van Vliet, C. W. N. Damen, F. M. van der Kloet, R. J. Vreeken, and T. Hankemeier, "Matrix effect compensation in small-molecule profiling for an LC-TOF platform using multicomponent postcolumn infusion," Analytical Chemistry, vol. 87, no. 12, pp. 5921-5929, 2015.

[28] E. Pagliano, Z. Mester, and J. Meija, "Calibration graphs in isotope dilution mass spectrometry," Analytica Chimica Acta, vol. 896, pp. 63-67, 2015.

[29] K. Habler, M. Gotthardt, J. Schüler, and M. Rychlik, "Multimycotoxin stable isotope dilution LC-MS/MS method for Fusarium toxins in beer," Food Chemistry, vol. 218, pp. 447-454, 2017.

[30] L. Qin, Y.-Y. Zhang, X.-B. Xu et al., "Isotope dilution HPLCMS/MS for simultaneous quantification of acrylamide and 5hydroxymethylfurfural (HMF) in thermally processed seafood," Food Chemistry, vol. 232, pp. 633-638, 2017.

[31] L. Wang, R. B. Cardenas, and C. Watson, "An isotope dilution ultra high performance liquid chromatography-tandem mass spectrometry method for the simultaneous determination of sugars and humectants in tobacco products," Journal of Chromatography A, vol. 1514, pp. 95-102, 2017.

[32] S. Yong, H. Liu, L. Wong et al., "Liquid chromatographyisotope dilution tandem mass spectrometry method for the measurement of urea in human serum and assignment of reference values to external quality assessment samples," International Journal of Mass Spectrometry, vol. 414, pp. 8793, 2017.

[33] T.-T. Feng, J.-H. Wu, X. Liang, M. Du, L. Qin, and X.-B. Xu, "Isotope dilution determination for the trace level of 4(5)methylimidazole in beverages using dispersive liquid-liquid microextraction coupled with ESI-HPLC-MS/MS," Food Chemistry, vol. 245, pp. 687-691, 2018.

[34] Y.-R. Lin, M.-F. Huang, Y.-Y. Wu et al., "Reductive amination derivatization for the quantification of garlic components by isotope dilution analysis," Food Chemistry, vol. 230, pp. 1-5, 2017.

[35] J. F. Bienvenu, G. Provencher, P. Bélanger et al., "Standardized procedure for the simultaneous determination of the matrix effect, recovery, process efficiency, and internal standard association," Analytical Chemistry, vol. 89, pp. 7560-7568, 2017.

[36] A. Ćirić, H. Prosen, M. Jelikić-Stankov, and P. Đurđević, "Evaluation of matrix effect in determination of some bioflavonoids in al food samples by LC-MS/MS method," Talanta, vol. 99, pp. 780-790, 2012.

[37] Ministry of Agriculture and Rural Affairs of the People's Republic of China, Ministry of Agriculture Announcement No.781-12-2006 Standard. 